\title{
Especies de Lutzomyia (Diptera: Psychodidae) posibles vectores de leishmaniasis en la ciudad de Bucaramanga, Santander, Colombia
}

\author{
Claudia M. Sandoval ${ }^{1}$, Víctor M. Angulo ${ }^{1}$, Reynaldo Gutiérrez ${ }^{1}$, Gerardo Muñoz ${ }^{1}$, Cristina Ferro ${ }^{2}$
}

\begin{abstract}
Resumen
Debido al registro, en los últimos cinco años, de ciento venticinco casos urbanos de leishmaniasis cutánea en la ciudad de Bucaramanga, se desarrolló el presente estudio con el fin de determinar las especies del género Lutzomyia existentes en la ciudad y sus implicaciones en salud pública. La búsqueda de los flebotomíneos se hizo en ocho zonas de la ciudad en los meses de septiembre a diciembre de 1996, utilizando como técnicas de muestreo trampas de luz tipo CDC y aspiración directa en cebo humano protegido y en sitios de reposo. Los insectos fueron preservados en alcohol al $70 \%$ y aclarados con $\mathrm{KOH}$ y fenol. Se capturaron 212 especímenes pertenecientes a once especies del género Lutzomyia y una del género Brumptomyia. Entre las especies antropofílicas encontradas, $L$. ovallesi y $L$. gomezi se destacan por ser reconocidas como vectores de Leishmania braziliensis. L. ovallesi fue la especie de mayor distribución en la ciudad, L. gomezi la más abundante en las capturas con cebo humano; las dos se encontraron en barrios donde se ha informado el mayor número de casos de la enfermedad. El hallazgo de estas especies en la ciudad sugiere la posibilidad de que los habitantes de Bucaramanga puedan infectarse en el área urbana y crea la necesidad de continuar estudios que contribuyan a interrumpir el ciclo de transmisión.
\end{abstract}

Lutzomyia species (Diptera: Psychodidae) possible leishmaniasis vector in the city of Bucaramanga, Santander, Colombia

One hundred and twenty five urban cases of cutaneous leishmaniasis have been reported within the city of Bucaramanga in the last five years. This study was carried out to determine the species of Lutzomyia present in the city and their role in public health. The search for sand flies was carried out in eight of the city's zones, from September to December 1996, using CDC light traps and direct aspiration in resting sites and on protected human bait. 212 specimens from eleven Lutzomyia species and one species of Brumptomyia were collected. Amongst the anthropophillic species found, $L$. ovallesi and $L$. gomezi are known vectors of $L$. braziliensis. $L$. ovallesi had the greatest distribution throughout the city and $L$. gomezi was the most frequent capture with human bait. Both were found in the dwellings from which most cases of the disease were reported. The finding of these species in the city suggests the possibility of infection within the urban area and the need to pursue studies to cut the transmission cycle.

${ }^{1}$ Centro de Investigaciones en Enfermedades Tropicales, CINTROP, UIS, Bucaramanga, Colombia.

${ }^{2}$ Laboratorio de Entomología, Instituto Nacional de Salud, Santa Fe de Bogotá, Colombia.

Recibido para su publicación: 2 de marzo de 1998. - Aceptado para su publicación: 22 de junio de 1998. 
Con el nombre de leishmaniasis se designa un complejo de enfermedades causadas por parásitos protozoarios de la familia Trypanosomatidae, género Leishmania, los cuales producen en humanos una gran variedad de manifestaciones clínicas (cutánea, mucocutánea, cutánea difusa y visceral) (1). Todas las leishmaniasis son transmitidas al hombre únicamente por la picadura de especies de flebótomos que, en el Nuevo Mundo, pertenecen al género Lutzomyia y en el Viejo Mundo al género Phlebotomus. En América, la leishmaniasis es endémica en muchas áreas tropicales y subtropicales y constituyen un grave problema de salud pública. La mayoría de casos humanos ocurre en personas que residen en áreas rurales o tienen contacto con hábitats selváticos ya que la enfermedad es básicamente una zoonosis. Cada especie de Leishmania tiene un único perfil epidemiológico con diferentes vectores, huéspedes reservorios y distribución geográfica (1-4).

La importancia de los cambios ecológicos y demográficos en la epidemiología de algunas leishmaniasis del Nuevo Mundo ha sido apreciada sólo recientemente. La epidemiología de estas enfermedades es extremadamente compleja y puede ser alterada por cambios en algún punto del triángulo epidemiológico que está formado por humanos, reservorios y los flebótomos transmisores. La mayoría de los factores ambientales (destrucción del bosque, nuevos asentamientos humanos, inundaciones, sequías, etc.) que afectan la epidemiología de varias leishmaniasis son aún pobremente entendidos o no se conocen muy bien; sin embargo, los datos disponibles sugieren que algunos de los parásitos y sus vectores pueden adaptarse a los cambios ecológicos tales como la deforestación y la urbanización. Cuando parásitos y vectores se adaptan a las condiciones alteradas, la epidemiología de la enfermedad asociada a estos cambios también cambia (5-7).

Para el caso de la leishmaniasis cutánea, el desarrollo urbano en áreas endémicas crea condiciones de interdigitación o superposición en donde la vivienda humana se aproxima a los fo- cos naturales con la consecuente aparición de individuos infectados (8). Aunque los primeros registros de casos urbanos de leishmaniasis proceden del estado de Rio de Janeiro, Brasil, de 1922, es en las últimas décadas que el informe de éstos ha venido en aumento (8-14). Sin embargo, hoy en día, la prevalencia de la leishmaniasis urbana todavía es considerablemente más baja que la estimada para el ambiente rural, pero no deja de ser un importante problema de salud pública.

En Colombia, infortunadamente, no se cuenta con un registro preciso de los primeros casos de leishmaniasis urbana, pero, antes de la década de los ochenta, la enfermedad solamente se asociaba con las zonas rurales y selváticas. Hasta la fecha, se han registrado casos de leishmaniasis urbana en los municipios de Durania, Norte de Santander, donde la infección urbana fue similar a la rural; en Remedios, Antioquia, se evidenció un foco de transmisión en dos barrios de la cabecera municipal (12); en Villeta, Cundinamarca, se presentaron cuatro casos urbanos en 1995 (13); en Leticia, Amazonas, entre 1992 y 1995, se registraron 25 casos aparentemente urbanos (14).

En la ciudad de Bucaramanga, Santander, en 1991 se registraron los primeros casos que motivaron la primera pesquisa entomológica en la que se encontraron las especies $L$. ovallesi, $L$. venezuelensis y L. shannoni (15). En el período comprendido entre 1992 y 1996 , se diagnosticaron 36 casos de leishmaniasis cutánea (16). Con estos antecedentes epidemiológicos, la alcaldía de Bucaramanga y el Centro de Investigaciones en Enfermedades Tropicales, con la asesoría del Instituto Nacional de Salud, decidieron conjuntamente implementar este estudio que tuvo como objetivos determinar las especies de Lutzomyia, sus relaciones con las características ecológicas de la ciudad y el papel que éstas desempeñan en la transmisión de la enfermedad en la meseta de Bucaramanga.

\section{Materiales y métodos}

Descripción del área. El estudio se desarrolló en zonas boscosas reforestadas, aledañas a los 
barrios periféricos de Bucaramanga, capital del departamento de Santander. La ciudad está ubicada en la meseta del mismo nombre, sobre la cordillera oriental, a $07^{\circ} 07^{\prime} 47^{\prime \prime}$ de latitud norte y $73^{\circ} 07^{\prime} 33^{\prime \prime}$ de longitud oeste y una altura sobre el nivel del mar entre los 900 y los $1.000 \mathrm{~m}$. El noroeste de la ciudad está bordeado por dos reservas forestales protectoras o escarpas denominadas occidental y de Malpaso, con una extensión aproximada de 3.169 hectáreas.

Debido a problemas de erosión, los bordes de la meseta están siendo reforestados desde 1967 por la Corporación de Defensa de la Meseta de Bucaramanga. En algunas partes, la vegetación se ha estabilizado hasta llegar a un bosque secundario, pero, en su mayor parte dominan especies arbustivas de una altura de $3 \mathrm{~m}$ y árboles esparcidos que alcanzan tamaños de 20 a $25 \mathrm{~m}$ con copas amplias. Las especies vegetales más abundantes son: Anacardium excelsum (caracolí), Schyzollobium parahybum (tambor), Cedrela odorata (cedro), Cecropia peltata (yarumo, orumo), Ficus glabrata (higuerón), Pseudosamanea guachapele (nauno), Bauhinia sp. (patevaca) y algunas especies faunísticas como Didelphis marsupialis (fara), Tamandua tetradactyla (oso hormiguero), Dasypus novemcintus (armadillo) Agouti paca (tinajo), Dacyprocta punctata (ñeque) e Iguana iguana (17).

Sitios de muestreo. Se seleccionaron ocho sitios de muestreo teniendo en cuenta características ecológicas como vegetación abundante o presencia de bosque secundario, preferiblemente, aledaños al lugar de procedencia de los casos de leishmaniasis registrados en el período comprendido entre 1992 y 1996.

Sitio 1 , bosque Chorreras; 2 , bosque Cinal; 3 , bosque Provenza; 4, La Rosita; 5, Terrazas; 6, parque Morrorrico; 7, UIS; 8, vivero Nazareth. (figura1)

Técnicas de muestreo. La búsqueda de los especímenes del género Lutzomyia se hizo de septiembre a diciembre de 1996, utilizando trampas CDC y aspiración directa sobre cebo

Figura 1. Aparición de casos de leishmaniasis cutanea en la meseta de Bucaramanga entre 1992 y 1997.

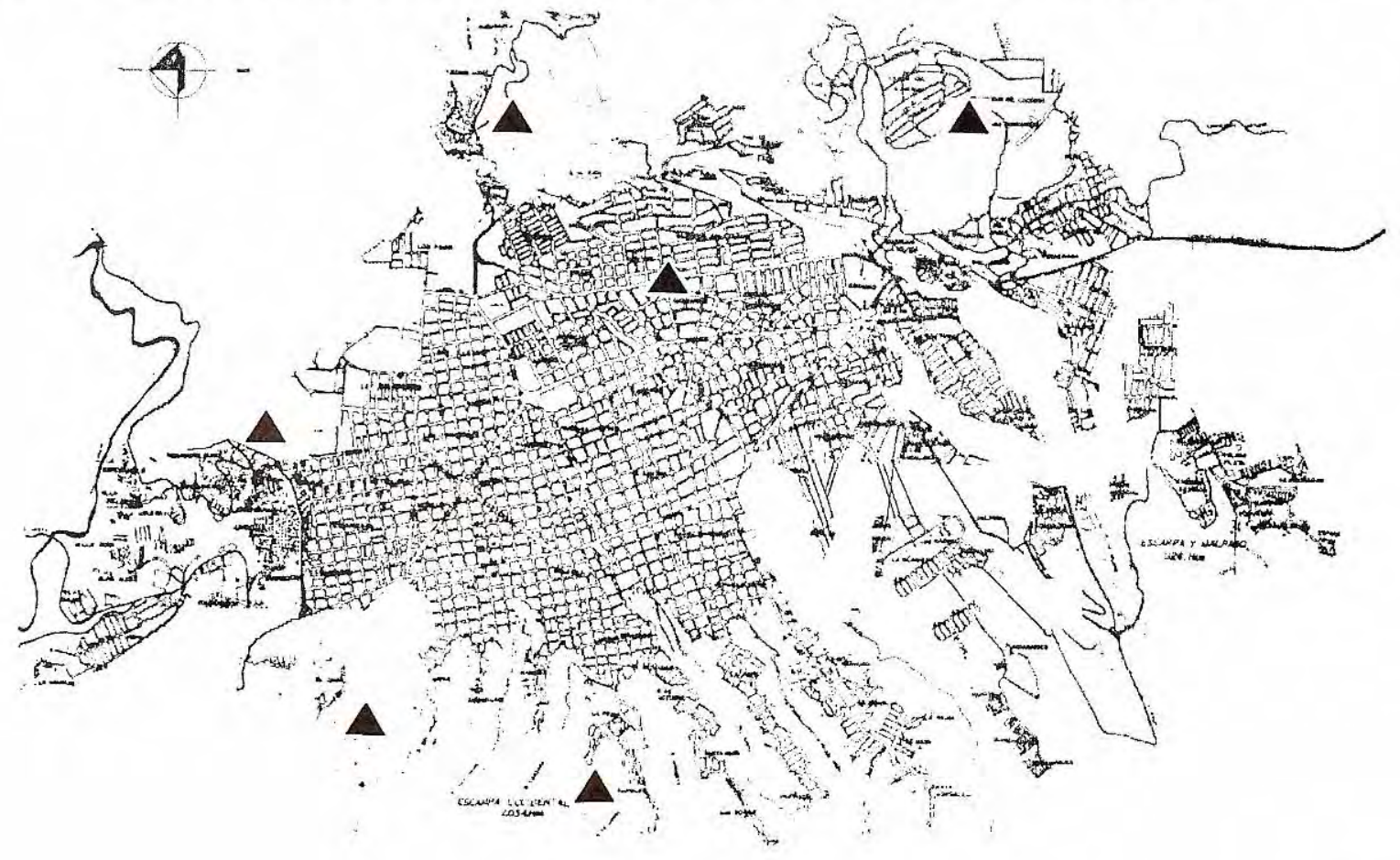


humano protegido, de la siguiente forma: durante dos noches consecutivas en cada uno de los sitios, cuatro personas, entre las 18:00 y 21:00 horas, realizaron las capturas en cebo humano y en las mismas noches se activaron tres trampas CDC, instaladas a una altura de 3 $\mathrm{m}$, entre las 18:00 y las 06:00 horas. Además, se realizaron capturas esporádicas en lugares de reposo como raíces y troncos de árbol en los sitios No. 1, 4 y 8.

Procesamiento e identificación de especímenes. Los insectos capturados fueron incluidos en alcohol al $70 \%$ y en el laboratorio se aclararon en $\mathrm{KOH}$ y fenol. Se realizaron montajes permanentes en una mezcla de bálsamofenol para posterior identificación mediante la aplicación de la clave taxonómica de Young y Duncan, 1994.

\section{Análisis estadístico}

Para agrupar los sitios de muestreo con base en la composición de especies, se utilizó la prueba de cluster analysis; como paso intermedio para determinar la similaridad entre pares de sitios de muestreo, se utilizó el índice de Jaccard y como técnica de agrupamiento, el promedio no ponderado. Se consideraron como sitios similares los que compartían, al menos, el $50 \%$ de las especies.

\section{Resultados}

Se realizaron diecisiete muestreos en ocho puntos de la ciudad, capturándose un total de 212 especímenes pertenecientes a once especies de Lutzomyia y una del género Brumptomyia. (cuadro 1). La riqueza de especies según el sitio de muestreo se distribuyó así: Vivero Nazaret con ocho especies de Lutzomyia, seguido del bosque Chorreras con seis, el bosque Cinal con cinco, el bosque Provenza y el parque Morrorrico con cuatro, La Rosita con tres, UIS y Terrazas, con una especie (cuadro 2). El método de captura más eficiente fue la trampa CDC en la cual el $92 \%$ de las especies estuvieron presentes (cuadro 3). La prueba de agrupamiento mostró similitud en la composición de especies entre los sitios vivero Nazaret y Provenza y entre el bosque Chorreras y La Rosita (figura1).

Cuadro 1. Flebótomos capturados en la ciudad de Bucaramanga.

\begin{tabular}{l|c|c|c|c|c|}
\cline { 4 - 6 } \multicolumn{1}{c|}{} & \multicolumn{3}{c|}{ METODOS DE MUESTREO } \\
\hline \multicolumn{1}{r|}{ ESPECIE } & HEMBRAS & MACHOS & CDC & CEBO HUMANO & REPOSO \\
\hline L. camposi & 28 & 31 & 9 & 0 & 50 \\
\hline L. dubitans & 13 & 50 & 6 & 0 & 57 \\
\hline L. gomezi & 15 & 4 & 6 & 13 & 0 \\
\hline L. ovallesi & 13 & 3 & 11 & 5 & 0 \\
\hline L. saulensis & 7 & 3 & 3 & 1 & 6 \\
\hline L. quasitownsendi & 7 & 4 & 5 & 6 & 0 \\
\hline L.shannoni & 2 & 8 & 1 & 2 & 7 \\
\hline L. walkeri & 1 & 2 & 1 & 0 & 2 \\
\hline L. venezuelensis & 0 & 3 & 0 & 0 & 3 \\
\hline L lichyi & 2 & 0 & 1 & 1 & 0 \\
\hline L. sordelli & 1 & 0 & 0 & 1 & 0 \\
\hline g. Brumptomyia & 11 & 4 & 16 & 0 & 0 \\
\hline TOTAL & 100 & 112 & 59 & 28 & 125 \\
\hline
\end{tabular}

Cuadro 2. Distribución y riqueza de especies en los sitios de muestreo.

\begin{tabular}{|c|c|c|c|c|c|c|c|c|c|c|c|c|c|c|}
\hline No. & SITIO DE MUESTREO & shannoni & quastownsendi & camposi & dubitans & sordellif & gomezi & \begin{tabular}{|l|} 
ovallesi \\
\end{tabular} & saulensis & walkeri & venezuelensis & tichyi & Brumptomyia & Riqueza \\
\hline 1 & Bosque chorreras & 1 & 1 & 1 & 1 & & & & 1 & 1 & & & & 6 \\
\hline 2 & Bosque cinal & & & 1 & 1 & & & 1 & & 1 & & & 1 & 5 \\
\hline 3 & Bosque provenza & & & 1 & & & 1 & 1 & 1 & & & & & 4 \\
\hline 4 & Bosque terrazas & & 1 & & & & & & & & & & & 1 \\
\hline 5 & La rosita CDMB & & & 1 & 1 & & & & 1 & & & & & 3 \\
\hline 6 & Parque morrorrico & & 1 & & & & 1 & 1 & & & & 1 & & 4 \\
\hline 7 & UIS & & & & & & & 1 & & & & & & 1 \\
\hline 8 & Vivero nazareth & & & 1 & 1 & 1 & 1 & 1 & 1 & & 1 & & 1 & 8 \\
\hline
\end{tabular}

Cuadro 3. Eficiencia por método de captura.

\begin{tabular}{|c|c|c|c|c|c|c|c|c|c|c|c|c|c|}
\hline METODO DE MUESTREO & shannoni & quasitownsendi & camposi & dubitans & sordelli & gomezi & ovallesi & sauiensis & walkeri & venezuelensis & lichyi & Brumptomyia & riqueza \\
\hline TRONCO DE ARBOL & 1 & & 1 & 1 & & & & 1 & 1 & 1 & & & 6 \\
\hline $\operatorname{cDC} 3 \mathrm{mts}$ & 1 & 1 & 1 & 1 & 1 & 1 & 1 & 1 & 1 & & 1 & 1 & 11 \\
\hline CEBO HUMANO & 1 & 1 & & & & 1 & 1 & 1 & & ( & 1 & & 6 \\
\hline
\end{tabular}




\section{Discusión}

En el presente estudio, aunque el número de especímenes capturados fue pequeño, se observó gran variedad de especies del género Lutzomyia, aumentando de 3 a 11 el número de especies registradas para la meseta de Bucaramanga en una zona altamente urbanizada, con pequeñas manchas de bosque secundario en recuperación y con el consecuente restablecimiento de la fauna. De las once especies de Lutzomyia capturadas, se destacan por su carácter antropofílico y sus antecedentes como vectores, L. ovallesi, L. gomezi, L. quasitownsendi, L. lichyi y L. shannoni.

L.ovallesi es vector confirmado de $L$. braziliensis en Guatemala y Venezuela $(18,19)$. En Bélice, Colombia y Panamá se ha encontrado con flagelados no identificados $(2,20,21)$. En este estudio, $L$. ovallesi fue la especie más ampliamente distribuida en el área, capturada en la mayoría de los sitios muestreados 2, 3, 6, 7 y 8 (mapa 1), algunos de éstos ubicados en los barrios El Cinal, San Rafael, Chorreras de Don Juan, que han informado gran número de casos de leishmaniasis. En algunas áreas de estos barrios, el nivel socioeconómico es bajo y son frecuentes las urbanizaciones de invasión próximas a áreas de bosque que bordean la ciudad.

De manera accidental se capturó un ejemplar dentro de una vivienda del barrio Chorreras de Don Juan, aledaña al bosque Chorreras, picando sobre humano, lo que refleja sus hábitos endofílicos y antropofílicos como se ha descrito en otros estudios. Por su amplia distribución, sus antecedentes como vector y sus hábitos antropofílicos, esta especie podría estar implicada en la transmisión de leishmaniasis cutánea en la ciudad.

Mapa 1. Sitios de muestreo en la ciudad de Bucaramanga.

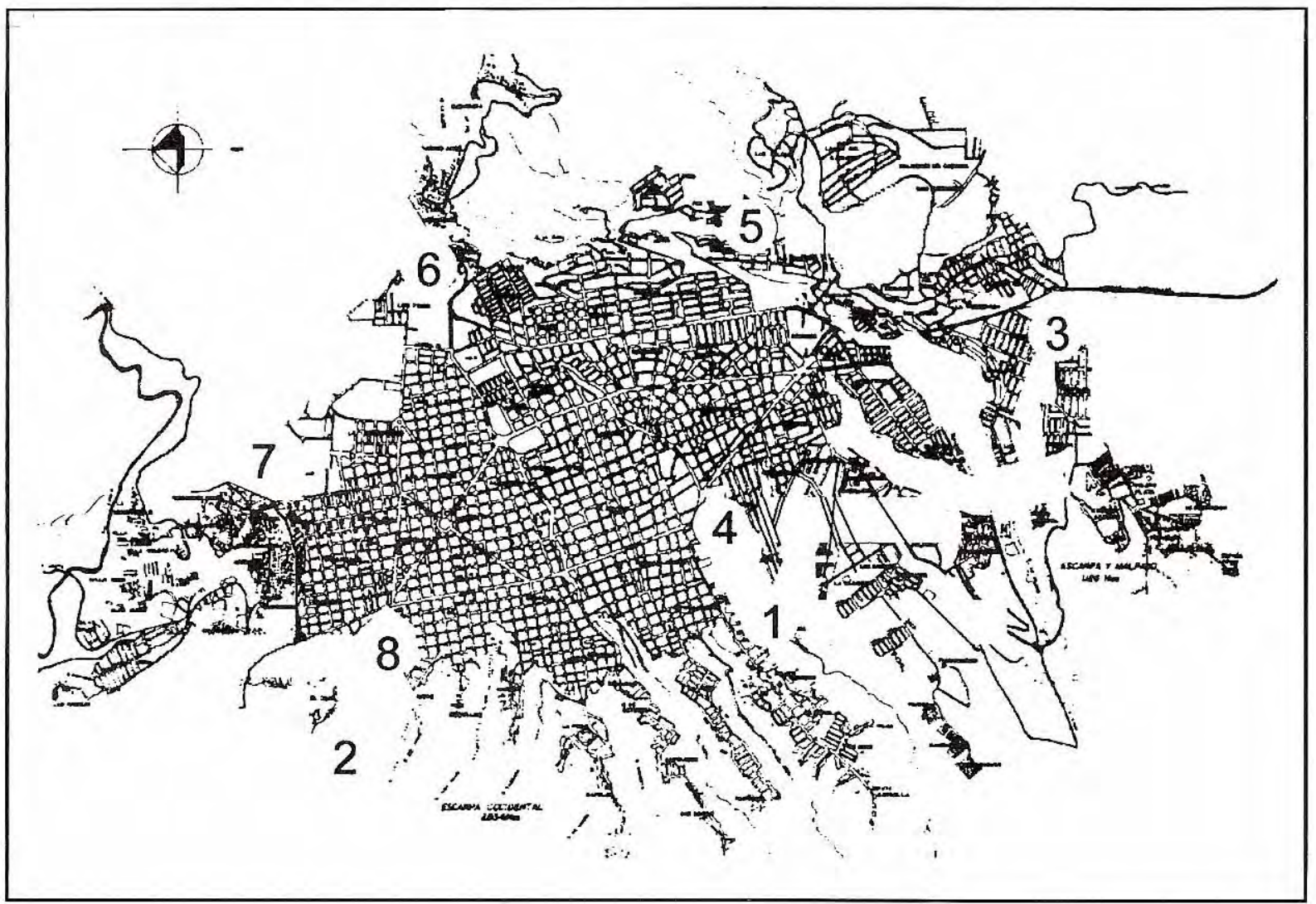


L. gomezi es una especie altamente antropofílica, aunque pica a una gran variedad de mamíferos; ha sido encontrada naturalmente infectada por L. braziliensis en Venezuela (19). En Colombia, esta especie tiene una distribución muy amplia y es frecuente en focos de leishmaniasis cutánea y visceral $(22,23)$, además, en barrios periféricos de algunas ciudades (15). En la meseta de Bucaramanga, fue la especie que más veces se capturó con cebo humano y, al igual que $L$. ovallesi, fue hallada en sitios donde se han registrado casos; no tuvo una distribución homogénea dentro del muestreo, pero se encontró en los sitios 3, 6 y 8 , los cuales enmarcan la ciudad.

L. quasitownsendi, especie que pertenece a la serie townsendi, la cual es importante en salud pública porque la mayoría de las hembras son altamente antropofílicas y varias de las especies que la forman son consideradas vectores sospechosos de leishmaniasis cutánea (2). Ha sido encontrada infectada con flagelados no identificados en ejemplares procedentes de la vereda Peñas Blancas, municipio de Landázuri, Santander (Muñoz G, tesis). Se encontró en los sitios 1,4 y 6 (mapa 1).

L. shannoni, especie que se ha encontrado infectada naturalmente con flagelados no identificados $(2,21)$ y es vector confirmado de estomatitis vesicular (24). En Colombia, es muy abundante en capturas en tronco de árbol (22) y, también, se ha encontrado en el peridomicilio (13). Es menos probable que esté involucrada en la transmisión.

L. lichyi ha sido considerado vector potencial de $L$. braziliensis, ya que posee un fuerte carácter antropofílico y, en laboratorio, ha desarrollado altas tasas de infección (25); fue capturada en un estudio realizado en el área urbana y rural de Villeta, Cundinamarca, en el que se plantea que esta especie puede tener un papel importante en la transmisión por su presencia en el peridomicilio (13). Accidentalmente, en este estudio, se capturó en intradomicilio un ejemplar de esta especie picando sobre humano; aunque es poco probable, no se descarta que esté involucrada en la transmisión.
Algunos de los sitios seleccionados en el estudio están localizados en zonas altamente reforestadas y continuas denominadas escarpas, los cuales presentan una composición de flora y fauna semejantes; esta similitud también se vio reflejada en la entomofauna, en este estudio, donde se observó que algunos sitios comparten el $50 \%$ de las especies de Lutzomyia (figura 1).

Por otro lado, también es importante tener en cuenta que la parte sur de la ciudad está avanzando hacia los bosques naturales, donde los factores de deforestación y urbanización crean condiciones de interdigitación o superposición en donde la vivienda humana se aproxima a los focos naturales (9), aumentando el riesgo de adquirir la enfermedad. Esta condición no se tuvo en cuenta en este estudio. Sin embargo, considerando que en otras ciudades del país no reforestadas se están presentando casos urbanos de leishmaniasis $(13,14)$, otros cambios ecológicos y demográficos pueden estar relacionados con la transmisión urbana.

En conclusión, la presencia de $L$. ovallesi, $L$. gomezi y $L$. quasitownsendi en la ciudad sugiere que los casos urbanos de leishmaniasis, notificados en Bucaramanga, sean autóctonos de la ciudad y que la reforestación ha aumentado el contacto de los habitantes con los vectores responsables de la transmisión de la enfermedad, especialmente, los de las viviendas ubicadas cerca a los bosques. Se recomienda realizar estudios relacionados con la biología de estas especies de Lutzomyia con antecedentes vectoriales para determinar la variación de la población flebotomínea durante las estaciones, sus hábitos intradomiciliarios y la presencia del parásito en los vectores.

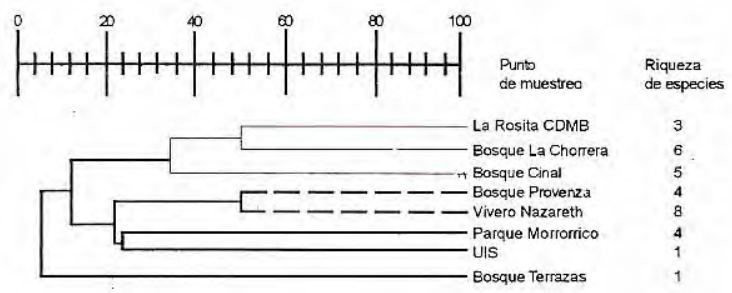

Figura 1. Prueba de Cluster. Agrupamiento de las estaciones de acuerdo a la composición de especies. 
Además, hacer un reconocimiento de la fauna de flebótomos en la parte de la ciudad no muestreada para tener una visión más amplia de los factores involucrados en la aparición de los casos urbanos, datos necesarios para desarrollar un programa adecuado de control de leishmaniasis en Bucaramanga.

\section{Agradecimientos}

Los autores agradecen a la alcaldía de Bucaramanga el apoyo financiero, la colaboración de la Corporación de Defensa de la Meseta de Bucaramanga (CDMB); al doctor Alberto Díaz por su apoyo en el manejo estadístico de los datos y por el apoyo técnico prestado por el señor Héctor Ariza.

\section{Referencias}

1. Desjeux P. Human leishmaniases: epidemiology and public health aspects. World Health Health Statist Quart 1992;45:267-75.

2. Young DG, Duncan M. Guide to the identification and geographic distribution of Lutzomyia sand flies in Mexico, the West Indies, Central and South America (Diptera: Psychodidae). Mem Amer Entomol Inst 1994;(54):1-881.

3. Tesh RB, Guzman H. Sandflies and the agents they transmit. En: The biology of disease vectors. University Press of Colorado; 1996. p. 117-27.

4. Grimaldi GJR, Tesh RB. Leishmaniasis of the New World: current concepts and implications for future research. Clin Microbiol Rev 1993;6:230-50.

5. Lainson R, Shaw JJ. Evolution, clasification and geografical distribution. In: Peters W, Killick-Kendrick, editors. The leishmaniasis in biology and medicine. London: Academic Press; 1987. p. 1:1-120.

6. Shaw JJ, Lainson R. Ecology and epidemiology: New World. In: Peters W, Killick-Kendrick, editors. The leishmaniasis in biology and medicine. London: Academic Press; 1987. p. 1:291-364.

7. Lainson R. Ecological interactions in the transmision of the leishmaniases. Phil Trans R Soc Lond 1988; 389-404.

8. Scorza JV, Macías P, Rojas J. Encuesta epidemiológica sobre leishmaniasis cutánea urbana en la ciudad de Trujillo, Venezuela. Bol Dir Mal San Amb 1985; 25:73-81.

9. Arias JR, Naiff R. The principal reservoir host of cutaneous leishmaniasis in the urban areas of Manaos, Central Amazon of Brasil.Mem Inst O Cruz 1981;76:279-86
10. Falqueto A, Coura JR, Barros GO, Grimaldi G, Lessa PA, Carias VR. Participacao do cao no ciclo de transmissao de leishmaniose tegumentar no municipio de Viana, Estado do Espirito Santo, Brasil. Mem Inst Oswaldo Cruz 1986;81:155-63.

11. Neto EA, Proenca N. Ocorrencia da leishmaniose tegumentar autoctone no municipio de Sao Paulo e adjacencias. Rev Paul Med 1960;56:374-9.

12. Ministerio de Salud. Leishmaniasis. Plan Nacional de Control. Normas técnico-administrativas; 1994.

13. Pardo RH, Farieta S, Munstermann LE, Ferro C. Estudio preliminar de los flebótomos de Villeta y Quebradanegra, Cundinamarca: sus implicaciones en salud pública. Biomédica 1996;16:293-302.

14. Ferro C, Pardo RH, Pérez L, Munstermann LE. Flebotomíneos de las áreas urbanas y rural de Leticia, Amazonas. Implicaciones en salud pública. Memorias, XVIII Congreso Socolen, 1996.

15. Ferro C, Morales A. Flebótomos de Colombia. Estudios realizados por el Laboratorio de Entomologia, INS, 1965-1997. En: Toro G, Hernández CA, Raad J, editores. Instituto Nacional de Salud 1917-1997: una historia, un compromiso. Santa Fe de Bogotá: Instituto Nacional de Salud; 1998.

16. Secretaría de Salud de Santander. Estadística de leishmaniasis, 1992-1997.

17. Suárez DJ. Estudio ecológico y ambiental de las reservas forestales protectoras de Bucaramanga. Tomo 1. Informe Corporacion de Defensa de la Meseta de Bucaramanga, 1995.

18. Rowton ED, De Mata M, Rizzo N, Porter CH, Navis TR. Isolation of Leishmania braziliensis from Lutzomyia ovallesi (Diptera: Psychodidae) in Guatemala. Am J Trop Med Hyg 1992;46:465-8.

19. Feliciangeli D, Rodríguez N, Bravo A. Vectors of cutaneous leishmaniasis in North-Central Venezuela. Med Veterin Entomol 1994;8:317-24.

20. Williams P. Phlebotominae sand flies and leishmaniasis in British Honduras (Belize). Trans R Soc Trop Med Hyg 1970;317-64.

21. Young DG, Morales A, Kreutzer RD, Alexander BJ, Corredor A, Tesh RB, et al. Isolations of Leishmania braziliensis (Kinetoplastida: Trypanosomatidae) from cryopreserved Colombian sand flies (Diptera: Psychodidae). J Med Entomol 1987;24(5): 587-9.

22. Alexander B, Ferro C, Young D, Morales A, Tesh RB. Ecology of flebotomine sandflies (Diptera: psychodidae) in a focus of Leishmania (Viannia) braziliensis in northeastern Colombia. Mem Inst Oswaldo Cruz 1992;83:387-95.

23. Ferro C, Morrison A, Torres M, Pardo R, Wilson ML, Tesh RB. Composition and relative abundance 
of sandflies of the genus Lutzomyia (Diptera: Psychodidae) at an endemic focus of visceral leishmaniasis in Colombia. J Med Entomol 1995;32(4): 527-37.

24. Comer JA, Tesh RB, Modi GB, Corn JL, Nettles VF. Vesicular stomatitis virus, New Jersey serotype: replication in and transmission by Lutzomyia shannoni (Diptera: Psychodidae). Am J Trop Med Hyg 1990;42:483-90.

25. Warburg A, Montoya-Lerma J, Jaramillo C, Cruz-Ruiz A, Ostrovska K. Leishmaniasis vector potencial of Lutzomyia spp. in Colombia coffee plantations. Med Vet Entomol 1995;5:9-16. 